\title{
Annular Bounds for Polynomial Zeros and Schur Stability of Difference Equations
}

\author{
Ke $\mathrm{Li}^{1}$ and Jin Liang ${ }^{2}$ \\ ${ }^{1}$ Shandong Key Laboratory of Automotive Electronic Technology, Institute of Automation, Shandong \\ Academy of Sciences, Jinan, Shandong 250014, China \\ 2 Department of Mathematics, Shanghai Jiao Tong University, Shanghai 200240, China
}

Correspondence should be addressed to Jin Liang, jinliang@sjtu.edu.cn

Received 7 October 2010; Accepted 30 October 2010

Academic Editor: Toka Diagana

Copyright (C 2011 K. Li and J. Liang. This is an open access article distributed under the Creative Commons Attribution License, which permits unrestricted use, distribution, and reproduction in any medium, provided the original work is properly cited.

\begin{abstract}
We investigate the monic complex-coefficient polynomial of degree $n, f(z):=z^{n}+a_{n-1} z^{n-1}+\cdots+a_{0}$ in the complex variable $z$ and obtain a new annular bound for the zeros of $f(z)$, which is sharper than the previous results and has clear advantages in judging the Schur stability of difference equations. In addition, examples are given to illustrate the theoretical result.
\end{abstract}

\section{Introduction}

It is well known that many discrete-time systems in engineering are described in terms of a difference equation, and the characteristic equation for the difference equation plays a key role in the study of the behaviors of the solutions, especially the stability of the solutions, to the discrete-time systems. Since the characteristic equations for difference equations are closely related to some complex polynomials, the estimates of the bound for the moduli of various complex polynomial zeros have been investigated by many researchers (cf. e.g., [18] and references therein). In the study on this issue, one of meaningful research ideas is to indicate such a common property of a lot of polynomials by a few very special polynomials. Using this idea, a good annular bound by estimating the largest nonnegative zeros of four specific polynomials is given in [8] recently. As a continuation of this work and our paper [4], in this paper we investigate further the location of the zeros of complex-coefficient polynomials on the basis of such a research idea and establish a new annular bound theorem (Theorem 3.1), which improves the previous corresponding result and has clear advantages in judging the Schur stability of difference equations. Examples are given to illustrate the advantages of the new result. 


\section{Preliminaries}

Throughout this paper, we let

$$
f(z):=z^{n}+a_{n-1} z^{n-1}+a_{n-2} z^{n-2}+\cdots+a_{1} z+a_{0}
$$

with $a_{i} \in \mathbf{C}, i \in\{0,1,2, \ldots, n-1\}$, and

$$
g(z):=(-1)^{n} f(z) f(-z)=z^{2 n}+b_{2 n-2} z^{2 n-2}+b_{2 n-4} z^{2 n-4}+\cdots+b_{2} z^{2}+b_{0} .
$$

Without losing the generality, we assume that $a_{0} \not \equiv 0$, or, equivalently, $b_{0} \not \equiv 0$.

Basic notations are as follows.

$\mathbf{R}_{-}:\{x \in \mathbf{R} \mid x<0\}$,

$|z|$ : the modulus of a complex number $z$,

$Z[f(z)]$ : the set of all zeros of $f(z)$,

$A[r, R]:\{z \in \mathbf{C}|r \leq| z \mid \leq R\}$ with $0 \leq r \leq R$,

$l$ : the smallest positive integer such that $a_{l} \neq 0$ in $f(z)$,

$k$ : the largest positive integer such that $a_{k} \neq 0$ in $f(z)$,

$q$ : the smallest positive integer such that $b_{2 q} \neq 0$ in $g(z)$,

$p$ : the largest positive integer such that $b_{2 p} \neq 0$ in $g(z)$,

$[m]$ : the integer part of a real number $m$.

In order to simplify the expressions in our study, we define specially that

$$
\sum_{i=t}^{s} y_{i}:=0
$$

for any positive integers $s, t(s<t)$, and sequence $\left\{y_{i} \in \mathrm{C}: s \leq i \leq t\right\}$. This notation is logical and useful in the note.

Moreover, we write

$$
c_{1}(x):=x^{n+l}+\sum_{i=n+1}^{n+l-1}\left|a_{i-l}\right| x^{i}+\sum_{i=2 l}^{n}\left|a_{i-l}-\frac{a_{0} a_{i}}{a_{l}}\right| x^{i}+\sum_{i=l+1}^{2 l-1} \frac{\left|a_{0} a_{i}\right|}{\left|a_{l}\right|} x^{i}-\frac{\left|a_{0}\right|^{2}}{\left|a_{l}\right|}
$$

with $a_{n}=1$ and $1 \leq l \leq[n / 2]$,

$$
c_{2}(x):=x^{2 n-k}-\sum_{i=k+1}^{n-1}\left|a_{i+k-n}\right| x^{i}-\sum_{i=n-k}^{k}\left|a_{i+k-n}-a_{i} a_{k}\right| x^{i}-\sum_{i=0}^{n-k-1}\left|a_{i} a_{k}\right| x^{i}
$$


with $[(n+1) / 2] \leq k \leq n-1$,

$$
d_{1}(x):=x^{2 n+2 q}+\sum_{i=n+1}^{n+q-1}\left|b_{2 i-2 q}\right| x^{2 i}+\sum_{i=2 q}^{n}\left|b_{2 i-2 q}-\frac{b_{0} b_{2 i}}{b_{2 q}}\right| x^{2 i}+\sum_{i=q+1}^{2 q-1} \frac{\left|b_{0} b_{2 i}\right|}{\left|b_{2 q}\right|} x^{2 i}-\frac{\left|b_{0}\right|^{2}}{\left|b_{2 q}\right|}
$$

with $b_{2 n}=1$ and $1 \leq q \leq[n / 2]$,

$$
d_{2}(x):=x^{4 n-2 p}-\sum_{i=p+1}^{n-1}\left|b_{2(i+p-n)}\right| x^{2 i}-\sum_{i=n-p}^{p}\left|b_{2(i+p-n)}-b_{2 i} b_{2 p}\right| x^{2 i}-\sum_{i=0}^{n-p-1}\left|b_{2 i} b_{2 p}\right| x^{2 i}
$$

with $[(n+1) / 2] \leq p \leq n-1$,

$$
\begin{array}{cc}
f_{1}(x):=x^{n}+\sum_{i=l}^{n-1}\left|a_{i}\right| x^{i}-\left|a_{0}\right|, & f_{2}(x):=x^{n}-\sum_{i=0}^{k}\left|a_{i}\right| x^{i}, \\
g_{1}(x):=x^{2 n}+\sum_{i=q}^{n-1}\left|b_{2 i}\right| x^{2 i}-\left|b_{0}\right|, & g_{2}(x):=x^{2 n}-\sum_{i=0}^{p}\left|b_{2 i}\right| x^{2 i} .
\end{array}
$$

Remark 2.1. By Descartes' rule of signs, it is easy to see that for each $i \in\{1,2\}$, the polynomial $c_{i}(x)\left(d_{i}(x), f_{i}(x), g_{i}(x)\right)$ has a unique positive zero.

We denote by $\alpha_{i}, \beta_{i}, \gamma_{i}$, and $\delta_{i}$ the unique positive zero of $c_{i}(x), d_{i}(x), f_{i}(x)$, and $g_{i}(x)$, respectively.

\section{Main Result}

The following result is established in [8].

Theorem A (see [8]). $Z[f(z)] \subset A[u, v]$, with $:=\max \left\{\gamma_{1}, \delta_{1}\right\}$ and $v:=\min \left\{\gamma_{2}, \delta_{2}\right\}$.

Theorem 3.1. Let $1 \leq l, q \leq[n / 2]$, and $[(n+1) / 2] \leq k, p \leq n-1$. Then

(i)

$$
Z[f(z)] \subset A[r, R]
$$

where $r:=\max \left\{\alpha_{1}, \beta_{1}\right\}$ and $R:=\min \left\{\alpha_{2}, \beta_{2}\right\}$

(ii)

$$
A[r, R] \subseteq A[u, v]
$$

where $u, v$ are constants as in Theorem A;

(iii) the annular bound of original polynomial $f(z)$ can be further improved by iterative procedure. 
Proof. Define

$$
\begin{aligned}
& \underline{c}(z):=f(z)\left(z^{l}-\frac{a_{0}}{a_{l}}\right) \\
& =z^{n+l}-\frac{a_{0}}{a_{l}} z^{n}+\sum_{i=l}^{n-1} a_{i} z^{i+l}-\sum_{i=l}^{n-1} \frac{a_{0} a_{i}}{a_{l}} z^{i}+a_{0} z^{l}-\frac{a_{0}^{2}}{a_{l}} \\
& =z^{n+l}+\sum_{i=2 l}^{n+l-1} a_{i-l} z^{i}-\sum_{i=l+1}^{n} \frac{a_{0} a_{i}}{a_{l}} z^{i}-\frac{a_{0}^{2}}{a_{l}} \\
& =z^{n+l}+\sum_{i=n+1}^{n+l-1} a_{i-l} z^{i}+\sum_{i=2 l}^{n} a_{i-l} z^{i}-\sum_{i=2 l}^{n} \frac{a_{0} a_{i}}{a_{l}} z^{i}-\sum_{i=l+1}^{2 l-1} \frac{a_{0} a_{i}}{a_{l}} z^{i}-\frac{a_{0}^{2}}{a_{l}} \\
& =z^{n+l}+\sum_{i=n+1}^{n+l-1} a_{i-l} z^{i}+\sum_{i=2 l}^{n}\left(a_{i-l}-\frac{a_{0} a_{i}}{a_{l}}\right) z^{i}-\sum_{i=l+1}^{2 l-1} \frac{a_{0} a_{i}}{a_{l}} z^{i}-\frac{a_{0}^{2}}{a_{l}}, \\
& \bar{c}(z):=f(z)\left(z^{n-k}-a_{k}\right) \\
& =z^{2 n-k}+\sum_{i=0}^{k-1} a_{i} z^{i+n-k}-\sum_{i=0}^{k} a_{i} a_{k} z^{i} \\
& =z^{2 n-k}+\sum_{i=n-k}^{n-1} a_{i+k-n} z^{i}-\sum_{i=0}^{k} a_{i} a_{k} z^{i} \\
& =z^{2 n-k}+\sum_{i=k+1}^{n-1} a_{i+k-n} z^{i}+\sum_{i=n-k}^{k}\left(a_{i+k-n}-a_{i} a_{k}\right) z^{i}-\sum_{i=0}^{n-k-1} a_{i} a_{k} z^{i}, \\
& \underline{d}(z):=g(z)\left(z^{2 q}-\frac{b_{0}}{b_{2 q}}\right) \\
& =z^{2 n+2 q}+\sum_{i=n+1}^{n+q-1} b_{2 i-2 q} z^{2 i}+\sum_{i=2 q}^{n}\left(b_{2 i-2 q}-\frac{b_{0} b_{2 i}}{b_{2 q}}\right) z^{2 i}-\sum_{i=q+1}^{2 q-1} \frac{b_{0} b_{2 i}}{b_{2 q}} z^{2 i}-\frac{b_{0}^{2}}{b_{2 q}}, \\
& \bar{d}(z):=g(z)\left(z^{2 n-2 p}-b_{2 p}\right) \\
& =z^{4 n-2 p}+\sum_{i=p+1}^{n-1} b_{2(i+p-n)} z^{2 i}+\sum_{i=n-p}^{p}\left(b_{2(i+p-n)}-b_{2 i} b_{2 p}\right) z^{2 i}-\sum_{i=0}^{n-p-1} b_{2 i} b_{2 p} z^{2 i},
\end{aligned}
$$

where $a_{n}=b_{2 n}=1$. Then it is not difficult to see that

$$
Z[f(z)] \subseteq Z[\underline{c}(z)] \cap Z[\bar{c}(z)] \cap Z[\underline{d}(z)] \cap Z[\bar{d}(z)]
$$

This implies that for every $w \in Z[f(z)]$ we have

$$
\underline{c}(w)=\bar{c}(w)=\underline{d}(w)=\bar{d}(w)=0,
$$


that is,

$$
\begin{gathered}
w^{n+l}+\sum_{i=n+1}^{n+l-1} a_{i-l} w^{i}+\sum_{i=2 l}^{n}\left(a_{i-l}-\frac{a_{0} a_{i}}{a_{l}}\right) w^{i}-\sum_{i=l+1}^{2 l-1} \frac{a_{0} a_{i}}{a_{l}} w^{i}-\frac{a_{0}^{2}}{a_{l}}=0, \\
w^{2 n-k}+\sum_{i=k+1}^{n-1} a_{i+k-n} w^{i}+\sum_{i=n-k}^{k}\left(a_{i+k-n}-a_{i} a_{k}\right) w^{i}-\sum_{i=0}^{n-k-1} a_{i} a_{k} w^{i}=0 \\
w^{2 n+2 q}+\sum_{i=n+1}^{n+q-1} b_{2 i-2 q} w^{2 i}+\sum_{i=2 q}^{n}\left(b_{2 i-2 q}-\frac{b_{0} b_{2 i}}{b_{2 q}}\right) w^{2 i}-\sum_{i=q+1}^{2 q-1} \frac{b_{0} b_{2 i}}{b_{2 q}} w^{2 i}-\frac{b_{0}^{2}}{b_{2 q}}=0 \\
w^{4 n-2 p}+\sum_{i=p+1}^{n-1} b_{2(i+p-n)} w^{2 i}+\sum_{i=n-p}^{p}\left(b_{2(i+p-n)}-b_{2 i} b_{2 p}\right) w^{2 i}-\sum_{i=0}^{n-p-1} b_{2 i} b_{2 p} w^{2 i}=0 .
\end{gathered}
$$

Hence, by (3.8), one has

$$
\begin{gathered}
\frac{\left|a_{0}\right|^{2}}{\left|a_{l}\right|} \leq|w|^{n+l}+\sum_{i=n+1}^{n+l-1}\left|a_{i-l}\right||w|^{i}+\sum_{i=2 l}^{n}\left|a_{i-l}-\frac{a_{0} a_{i}}{a_{l}}\right||w|^{i}+\sum_{i=l+1}^{2 l-1} \frac{\left|a_{0} a_{i}\right|}{\left|a_{l}\right|}|w|^{i}, \\
|w|^{2 n-k} \leq \sum_{i=k+1}^{n-1}\left|a_{i+k-n}\right||w|^{i}+\sum_{i=n-k}^{k}\left|a_{i+k-n}-a_{i} a_{k}\right||w|^{i}+\sum_{i=0}^{n-k-1}\left|a_{i} a_{k}\right||w|^{i}, \\
\frac{\left|b_{0}\right|^{2}}{\left|b_{2 q}\right|} \leq|w|^{2 n+2 q}+\sum_{i=n+1}^{n+q-1}\left|b_{2 i-2 q}\right||w|^{2 i}+\sum_{i=2 q}^{n}\left|b_{2 i-2 q}-\frac{b_{0} b_{2 i} \mid}{b_{2 q}}\right||w|^{2 i}+\sum_{i=q+1}^{2 q-1} \frac{\left|b_{0} b_{2 i}\right|}{\left|b_{2 q}\right|}|w|^{2 i}, \\
|w|^{4 n-2 p} \leq \sum_{i=p+1}^{n-1}\left|b_{2(i+p-n)}\right||w|^{2 i}+\sum_{i=n-p}^{p}\left|b_{2(i+p-n)}-b_{2 i} b_{2 p}\right||w|^{2 i}+\sum_{i=0}^{n-p-1}\left|b_{2 i} b_{2 p}\right||w|^{2 i},
\end{gathered}
$$

which imply that

$$
Z[f(z)] \subset\left\{z \in \mathbf{C}: c_{1}(|z|) \geq 0, d_{1}(|z|) \geq 0, c_{2}(|z|) \leq 0, d_{2}(|z|) \leq 0\right\} .
$$

In addition, it follows from (2.4)-(2.7) that

$$
\begin{array}{r}
c_{1}(x)<0, \quad \forall x \in\left[0, \alpha_{1}\right), \\
c_{1}(x) \geq 0, \quad \forall x \in\left[\alpha_{1},+\infty\right), \\
d_{1}(x)<0, \quad \forall x \in\left[0, \beta_{1}\right), \\
d_{1}(x) \geq 0, \quad \forall x \in\left[\beta_{1},+\infty\right),
\end{array}
$$




$$
\begin{gathered}
c_{2}(x) \leq 0, \quad \forall x \in\left[0, \alpha_{2}\right], \\
c_{2}(x)>0, \quad \forall x \in\left(\alpha_{2},+\infty\right), \\
d_{2}(x) \leq 0, \quad \forall x \in\left[0, \beta_{2}\right], \\
d_{2}(x)>0, \quad \forall x \in\left(\beta_{2},+\infty\right) .
\end{gathered}
$$

Therefore, for each $w \in Z[f(z)]$ we have

$$
|w| \geq \alpha_{1}, \quad|w| \geq \beta_{1}, \quad|w| \leq \alpha_{2}, \quad|w| \leq \beta_{2},
$$

which imply that (3.1) is hold. So (i) is proved.

Next we prove that (ii) holds. Actually, we have

$$
\begin{aligned}
& c_{1}\left(r_{1}\right)=r_{1}^{n+l}+\sum_{i=n+1}^{n+l-1}\left|a_{i-l}\right| r_{1}^{i}+\sum_{i=2 l}^{n}\left|a_{i-l}-\frac{a_{0} a_{i}}{a_{l}}\right| r_{1}^{i}+\sum_{i=l+1}^{2 l-1} \frac{\left|a_{0} a_{i}\right|}{\left|a_{l}\right|} r_{1}^{i}-\frac{\left|a_{0}\right|^{2}}{\left|a_{l}\right|} \\
& =\left(\left|a_{0}\right|-\sum_{i=l}^{n-1}\left|a_{i}\right| \gamma_{1}^{i}\right) \gamma_{1}^{l}+\sum_{i=n+1}^{n+l-1}\left|a_{i-l}\right| \gamma_{1}^{i}+\sum_{i=2 l}^{n}\left|a_{i-l}-\frac{a_{0} a_{i}}{a_{l}}\right| \gamma_{1}^{i}+\sum_{i=l+1}^{2 l-1} \frac{\left|a_{0} a_{i}\right|}{\left|a_{l}\right|} \gamma_{1}^{i}-\frac{\left|a_{0}\right|^{2}}{\left|a_{l}\right|} \\
& =\left|a_{0}\right| \gamma_{1}^{l}-\sum_{i=2 l}^{n+l-1}\left|a_{i-l}\right| \gamma_{1}^{i}+\sum_{i=n+1}^{n+l-1}\left|a_{i-l}\right| \gamma_{1}^{i}+\sum_{i=2 l}^{n}\left|a_{i-l}-\frac{a_{0} a_{i}}{a_{l}}\right| \gamma_{1}^{i}+\sum_{i=l+1}^{2 l-1} \frac{\left|a_{0} a_{i}\right|}{\left|a_{l}\right|} \gamma_{1}^{i} \\
& -\frac{\left|a_{0}\right|}{\left|a_{l}\right|}\left(r_{1}^{n}+\sum_{i=l}^{n-1}\left|a_{i}\right| r_{1}^{i}\right) \\
& =-\sum_{i=2 l}^{n}\left|a_{i-l}\right| \gamma_{1}^{i}+\sum_{i=2 l}^{n}\left|a_{i-l}-\frac{a_{0} a_{i}}{a_{l}}\right| \gamma_{1}^{i}+\sum_{i=l+1}^{2 l-1} \frac{\left|a_{0} a_{i}\right|}{\left|a_{l}\right|} \gamma_{1}^{i}-\sum_{i=l+1}^{n} \frac{\left|a_{0} a_{i}\right|}{\left|a_{l}\right|} \gamma_{1}^{i} \\
& =-\sum_{i=2 l}^{n}\left(\left|a_{i-l}\right|+\left|\frac{a_{0} a_{\mathrm{i}}}{a_{l}}\right|-\left|a_{i-l}-\frac{a_{0} a_{i}}{a_{l}}\right|\right) r_{1}^{i} \\
& \leq 0 \text {, }
\end{aligned}
$$

where $a_{n}=1$ and $1 \leq l \leq[n / 2]$.

On the other hand, since the polynomial equation $c_{1}(x)=0$ has a unique positive root $\alpha_{1}$ and

$$
\begin{gathered}
c_{1}(x) \leq 0, \quad \forall x \in\left[0, \alpha_{1}\right], \\
c_{1}(x)>0, \quad \forall x \in\left(\alpha_{1},+\infty\right),
\end{gathered}
$$

we get $\alpha_{1} \geq \gamma_{1}$ by combining(3.13) and (3.14). 
In addition, we have

$$
\begin{aligned}
c_{2}\left(\gamma_{2}\right) & =\gamma_{2}^{2 n-k}-\sum_{i=k+1}^{n-1}\left|a_{i+k-n}\right| \gamma_{2}^{i}-\sum_{i=n-k}^{k}\left|a_{i+k-n}-a_{i} a_{k}\right| \gamma_{2}^{i}-\sum_{i=0}^{n-k-1}\left|a_{i} a_{k}\right| \gamma_{2}^{i} \\
& =\sum_{i=0}^{k}\left|a_{i}\right| \gamma_{2}^{i+n-k}-\sum_{i=k+1}^{n-1}\left|a_{i+k-n}\right| \gamma_{2}^{i}-\sum_{i=n-k}^{k}\left|a_{i+k-n}-a_{i} a_{k}\right| \gamma_{2}^{i}-\sum_{i=0}^{n-k-1}\left|a_{i} a_{k}\right| \gamma_{2}^{i} \\
& =\sum_{i=n-k}^{n}\left|a_{i+k-n}\right| \gamma_{2}^{i}-\sum_{i=k+1}^{n-1}\left|a_{i+k-n}\right| \gamma_{2}^{i}-\sum_{i=n-k}^{k}\left|a_{i+k-n}-a_{i} a_{k}\right| \gamma_{2}^{i}-\sum_{i=0}^{n-k-1}\left|a_{i} a_{k}\right| \gamma_{2}^{i} \\
& =\left|a_{k}\right| \gamma_{2}^{n}+\sum_{i=n-k}^{k}\left|a_{i+k-n}\right| \gamma_{2}^{i}-\sum_{i=n-k}^{k}\left|a_{i+k-n}-a_{i} a_{k}\right| \gamma_{2}^{i}-\sum_{i=1}^{n-k-1}\left|a_{i} a_{k}\right| \gamma_{2}^{i}-\left|a_{0}\right|\left|a_{k}\right| \\
& =\left|a_{k}\right| \gamma_{2}^{n}+\sum_{i=n-k}^{k}\left(\left|a_{i+k-n}\right|-\left|a_{i+k-n}-a_{i} a_{k}\right|\right) \gamma_{2}^{i}-\sum_{i=1}^{n-k-1}\left|a_{i} a_{k}\right| \gamma_{2}^{i}-\left(\gamma_{2}^{n}-\sum_{i=1}^{k}\left|a_{i}\right| \gamma_{2}^{i}\right)\left|a_{k}\right| \\
& =\sum_{i=n-k}^{k}\left(\left|a_{i+k-n}\right|+\left|a_{i} a_{k}\right|-\left|a_{i+k-n}-a_{i} a_{k}\right|\right) \gamma_{2}^{i} \\
& \geq 0 .
\end{aligned}
$$

Since

$$
\begin{gathered}
c_{2}(x)<0, \quad \forall x \in\left[0, \alpha_{2}\right), \\
c_{2}(x) \geq 0, \quad \forall x \in\left[\alpha_{2},+\infty\right),
\end{gathered}
$$

we have $\alpha_{2} \leq \gamma_{2}$.

In the same way, we can obtain $\beta_{1} \geq \delta_{1}$ and $\beta_{2} \leq \delta_{2}$; therefore,

$$
A[r, R] \subseteq A[u, v] .
$$

Finally, we prove (iii). Set

$$
\underline{c}^{(1)}(z):=\underline{c}(z)=z^{n+l}+a_{n+l-1}^{(1)} z^{n+l-1}+\cdots+a_{l+1}^{(1)} z^{l+1}+a_{0}^{(1)},
$$

with

$$
a_{i}^{(1)}= \begin{cases}a_{i-l}, & n+1 \leq i \leq n+l-1 ; \\ a_{i-l}-\frac{a_{0} a_{i}}{a_{l}}, & 2 l \leq i \leq n ; \\ -\frac{a_{0} a_{i}}{a_{l}}, & l+1 \leq i \leq 2 l-1 ; \\ -\frac{a_{0}^{2}}{a_{l}}, & i=0,\end{cases}
$$


and let $l_{1}$ be the smallest positive integer such that $a_{l_{1}}^{(1)} \neq 0$ in $\underline{c}^{(1)}(z)$. If $l+1 \leq l_{1} \leq[(n+l) / 2]$, in analogy to (3.3) and (2.4), we can define

$$
\underline{c}^{(2)}(z):=\underline{c}^{(1)}(z)\left(z^{l_{1}}-\frac{a_{0}^{(1)}}{a_{l_{1}}^{(1)}}\right)
$$

and $c_{1}^{(2)}(x)$, respectively. It is not difficult to see that, the unique positive root of polynomial $c_{1}^{(2)}(x), \alpha_{1}^{(2)} \geq \alpha_{1}$. Similarly, we can define $c_{2}^{(2)}(x), d_{1}^{(2)}(x)$, and $d_{2}^{(2)}(x)$, respectively. Moreover, their respective positive roots $\alpha_{2}^{(2)}, \beta_{1}^{(2)}$, and $\beta_{2}^{(2)}$ satisfy that

$$
\alpha_{2}^{(2)} \leq \alpha_{2}, \quad \beta_{1}^{(2)} \geq \beta_{1}, \quad \beta_{2}^{(2)} \leq \beta_{2}
$$

Consequently, new annular bound of $f(z)$, namely, $A\left[r^{(2)}, R^{(2)}\right]$ with

$$
r^{(2)}:=\max \left\{\alpha_{1}^{(2)}, \beta_{1}^{(2)}\right\}, \quad R^{(2)}:=\min \left\{\alpha_{2}^{(2)}, \beta_{2}^{(2)}\right\}
$$

is better than (3.1). This procedure can be applied iteratively.

$$
c_{1}^{(2)}(x), \quad c_{2}^{(2)}(x), \quad d_{1}^{(2)}(x), \quad d_{2}^{(2)}(x)
$$

can be further transformed into

$$
c_{1}^{(3)}(x), \quad c_{2}^{(3)}(x), \quad d_{1}^{(3)}(x), \quad d_{2}^{(3)}(x),
$$

respectively, and

$$
c_{1}^{(3)}(x), \quad c_{2}^{(3)}(x), \quad d_{1}^{(3)}(x), \quad d_{2}^{(3)}(x)
$$

into

$$
c_{1}^{(4)}(x), \quad c_{2}^{(4)}(x), \quad d_{1}^{(4)}(x), \quad d_{2}^{(4)}(x),
$$

until the last iteration brings no practical improvement. Obviously, when $m$ increases,

$$
r^{(m)}\left(:=\max \left\{\alpha_{1}^{(m)}, \beta_{1}^{(m)}\right\}\right), \quad R^{(m)}\left(:=\min \left\{\alpha_{2}^{(m)}, \beta_{2}^{(m)}\right\}\right)
$$

will approach the smallest and largest modulus of polynomial zero, respectively, where

$$
\alpha_{1}^{(m)}\left(\operatorname{resp} \cdot \alpha_{2}^{(m)}, \beta_{1}^{(m)}, \beta_{2}^{(m)}\right)
$$


denotes the unique positive root of

$$
c_{1}^{(m)}(x)\left(\operatorname{resp} \cdot c_{2}^{(m)}(x), d_{1}^{(m)}(x), d_{2}^{(m)}(x)\right) .
$$

This means that (iii) is true.

Remark 3.2. (a) When $c_{2}(r)>0$, it follows from (3.14) and (3.15) that for every $w \in Z[f(z)]$, $|w| \leq \alpha_{2}<r$, that is, $Z[f(z)] \subset B(r)$, that is, $f(z)$ is $r$-stable.

Similarly, we can draw the same conclusion when $d_{2}(r)>0$, and $Z[f(z)] \subset \bar{B}^{c}(r)$ when $c_{1}(r)<0$ or $d_{1}(r)<0$.

(b) By the similar arguments in the proof of (iii) of Theorem 3.1, the results in (a) can be improved. This also provides an iterative algorithm to test the r-stability and Schur stability of polynomials.

(c) The question "What happens to Theorem 3.1 when $n-1 \geq l, q>[n / 2]$, and $1 \leq k, p<$ $[(n+1) / 2]$ ?" is worth considering further.

Example 3.3. Let

$$
f(z)=z^{3}+(1+j) z^{2}+2 j z+1
$$

where $j=\sqrt{-1}$. By Theorem 3.1, we obtain

$$
Z[f(z)] \subset A[0.389,1.647]
$$

If we start the iterative procedure given in the proof of (iii) of Theorem 3.1, after five iterations, we obtain

$$
Z[f(z)] \subset A[0.390,1.644]
$$

On the other hand, by Theorem A, one only can have

$$
Z[f(z)] \subset A[0.387,1.938]
$$

The following examples show the advantages of Theorems 3.1 over Theorem A in analyzing the Schur stability of difference equations (discrete-time systems).

Example 3.4. Let the characteristic polynomial of a difference equation (discrete-time system) be given by

$$
f(z)=z^{3}+\frac{1}{2}(\sqrt{2}+j) z^{2}+\left(\frac{1}{4}+\frac{\sqrt{2}}{2} j\right) z-\frac{1}{8}(\sqrt{2}-5 j)
$$


where $j=\sqrt{-1}$. Then by Theorem 3.1, we get $c_{2}(1)=7 / 16>0$, which implies that all zeros of $f(z)$ lie in the open unit disk, that is, this system is Schur stable. However, by Theorem A, one has

$$
Z[f(z)] \subset A[0.638,1.175]
$$

So Theorem A cannot guarantee the stability of such a system.

Example 3.5. Suppose the characteristic polynomial of a difference equation (discrete-time system) is given by

$$
f(z)=z^{3}+\left(\frac{1}{2}+j\right) z^{2}-\left(\frac{3}{4}-j\right) z-\left(\frac{11}{8}+\frac{1}{4} j\right)
$$

where $j=\sqrt{-1}$. Then by Theorem 3.1, we have $c_{1}(1)=-9 / 16<0$, which implies that all zeros of $f(z)$ are outside the open unit disk, namely, such a system is instable. By Theorem A, one has

$$
Z[f(z)] \subset A[0.824,1.517]
$$

which cannot determine the instability of this system.

Example 3.6. Consider the following characteristic polynomial of a difference equation (discrete-time system):

$$
f(z)=z^{4}+2 z^{3}+2 z^{2}+z+\sqrt{11}-3 .
$$

In this example,

$$
c_{2}^{(1)}=-6.316, \quad d_{2}^{(1)}=0 ; \quad c_{2}^{(2)}=-7.584, \quad d_{2}^{(2)}=0.203
$$

Consequently, such a difference equation (discrete-time system) is Schur stable.

\section{Acknowledgments}

This work was supported partially by the NSF of China (10771202) and the Specialized Research Fund for the Doctoral Program of Higher Education of China (2007035805).

\section{References}

[1] F. G. Boese and W. J. Luther, "A note on a classical bound for the moduli of all zeros of a polynomial," IEEE Transactions on Automatic Control, vol. 34, no. 9, pp. 998-1001, 1989.

[2] G. T. Cargo and O. Shisha, "Zeros of polynomials and fractional order differences of their coefficients," Journal of Mathematical Analysis and Applications, vol. 7, pp. 176-182, 1963.

[3] R. B. Gardner and N. K. Govil, "On the location of the zeros of a polynomial," Journal of Approximation Theory, vol. 78, no. 2, pp. 286-292, 1994. 
[4] J. Liang and K. Li, "A new stability criterion for polynomials with the quasi-critical condition," International Journal of Nonlinear Sciences and Numerical Simulation, vol. 9, no. 3, pp. 289-292, 2008.

[5] M. Marden, Geometry of Polynomials, Mathematical Surveys, No. 3, American Mathematical Society, Providence, RI, USA, 2nd edition, 1966.

[6] G. V. Milovanovic, D. S. Milovanovic, and T. M. Rassias, Topics in Polynomials, Extremal Problems, Inequalities, Zeros, World Scientic, Singapore, 1994.

[7] Q. I. Rahman and G. Schmeisser, Analytic Theory of Polynomials, Oxford University Press, Oxford, UK, 2005.

[8] Y.-J. Sun, "New result for the annular bounds of complex-coefficient polynomial zeros," IEEE Transactions on Automatic Control, vol. 49, no. 5, pp. 813-814, 2004. 